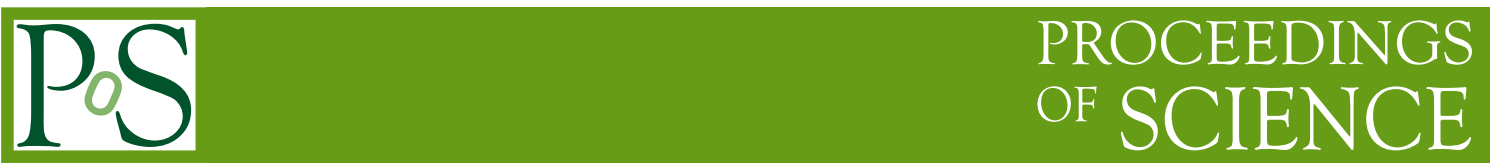

\title{
Quark number susceptibility of high temperature QCD
}

\section{Ari Hietanen*}

Theoretical Physics Division, Department of Physical Sciences P.O.Box 64 FI-00014 University of Helsinki, Finland and

Helsinki Institute of Physics, P.O.Box 64, FI-00014 University of Helsinki, Finland

E-mail: ari.hietanen@helsinki.fi

\section{Kari Rummukainen}

Department of Physics, University of Oulu P.O.Box 3000, FI-90014 Oulu, Finland and Department of Physics, Theory Division CERN CH-1211 Geneva, Switzerland

E-mail: kari.rummukainen@oulu.fi

We use three dimensional reduced effective field theory (EQCD) and lattice calculations to determine the quark number susceptibility of QCD at high temperature. We find our results to agree well with known perturbative expansion as well as with other lattice data.

XXIVrd International Symposium on Lattice Field Theory

25-30 July 2006

Tucson, Arizona, July 23-28, 2006

${ }^{*}$ Speaker. 


\section{Introduction}

A dimensionally reduced effective theory is, by now, a well-established and powerful tool for studying high-temperature QCD both perturbatively and non-perturbatively. At high enough temperatures the QCD coupling becomes small, and perturbative methods can be safely applied to hard $(\sim T)$ modes. The fact that high-temperature QCD can be reduced to $3 \mathrm{~d}$ effective theory can be understood by considering an Euclidean finite- $T$ propagator

$$
\frac{1}{p^{2}+\omega_{n}^{2}+m^{2}}
$$

where $\omega_{n}^{b}=2 n \pi T$ for bosons and $\omega_{n}^{f}=(2 n+1) \pi T$ for fermions. Thus, only static $n=0$ bosonic modes remain "light" at high temperatures, and the heavy $n \neq 0$ bosonic and all fermionic modes can be integrated perturbatively $[1,2,3,4]$. The result is an effective $3 \mathrm{~d}$ theory of full QCD, electrostatic QCD (EQCD) [11]. An essential feature of the effective theory is that it fully includes the perturbatively problematic soft $(\sim g T)$ and ultrasoft $\left(\sim g^{2} T\right)$ scales of the original theory; only perturbatively well-controlled hard scales $(\sim T)$ are integrated over.

EQCD offers a good starting point both for perturbative calculations $[5,6,7]$ and non-perturbative lattice simulations. In the latter case, EQCD offers an interesting alternative to standard hightemperature lattice simulations: above all, the effective theory is purely bosonic and only 3dimensional, making it much cheaper to simulate. The theory is superrenormalizable, which renders the continuum limit particularly transparent; it also enables simulations at arbitrarily large temperatures. On the other hand, the effective theory cannot be used to study the QCD phase transition: at too low $T$ QCD becomes strongly coupled and the perturbative derivation of EQCD fails. Nevertheless, the theory has been found to work well down to temperatures $T \sim 1.5-3 T_{c}$, depending on observable used. Lattice simulations of EQCD have been used to calculate QCD pressure at high T [8], spatial string tension [9], and spatial screening lengths [10]. Here we measure the quark number susceptibility, and compare the result to both perturbative and $4 \mathrm{~d}$ lattice results.

\section{Susceptibility in electrostatic QCD}

EQCD is defined by the action

$$
\begin{aligned}
& S_{\mathrm{E}}=\int \mathrm{d}^{3} x \mathscr{L}_{\mathrm{E}} \\
& \mathscr{L}_{\mathrm{E}}=\frac{1}{2} \operatorname{Tr}\left[F_{i j}^{2}\right]+\operatorname{Tr}\left[D_{i}, A_{0}\right]^{2}+m_{3}^{2} \operatorname{Tr}\left[A_{0}^{2}\right]+i \gamma_{3} \operatorname{Tr}\left[A_{0}^{3}\right]+\lambda_{3}\left(\operatorname{Tr}\left[A_{0}^{2}\right]\right)^{2},
\end{aligned}
$$

where $F_{i j}=\partial_{i} A_{j}-\partial_{j} A_{i}+i g_{3}\left[A_{i}, A_{j}\right]$ and $D_{i}=\partial_{i}+i g_{3} A_{i} . F_{i j}, A_{i}$ and $A_{0}$ are traceless $3 \times 3$ Hermitean matrices $\left(A_{0}=A_{0}^{a} T_{a}\right.$, etc). Coupling and mass parameters $g_{3}, m_{3}, \gamma_{3}$ and $\lambda_{3}$ are defined by the physical $4 \mathrm{~d}$ temperature, renormalization scale $\Lambda_{\overline{\mathrm{MS}}}$, chemical potential $\mu$ and the number of massless fermions. It is convenient to use the dimensionless ratios

$$
y=\frac{m_{3}^{2}}{g_{3}^{4}}, x=\frac{\lambda_{3}}{g_{3}^{2}}, z=\frac{\gamma_{3}}{g_{3}^{3}},
$$


which determine the physical properties of EQCD. The $\mu$-dependence of the parameters is, at 1loop level,

$$
y=y_{\mu=0}\left(1+\sum_{f} \bar{\mu}_{f}^{2} \frac{3}{2 N_{\mathrm{c}}+N_{\mathrm{f}}}\right), \quad z=\sum_{f} \frac{\bar{\mu}_{f}}{3 \pi}, \quad x=x_{\mu=0},
$$

where $\bar{\mu}=\mu /(\pi T)$ and the $\mu=0$ expressions can be found in ref. [11]. The two loop corrections have been calculated in ref. [10], but the effects remain in practice negligible.

The quantity we are interested in is the quark number susceptibility, which we calculate over one flavor $u$ only, can be defined in EQCD as

$$
\chi_{3}=\frac{1}{V} \frac{\partial^{2}}{\partial \mu_{u}^{2}} \ln \mathscr{Z}=\frac{1}{V} \frac{\partial^{2}}{\partial \mu_{u}^{2}} \ln \int \mathscr{D} A_{k} A_{0} \exp -S_{\mathrm{E}}
$$

Substituting $S_{\mathrm{E}}$ from (2.1) we arrive at equation

$$
\begin{aligned}
\chi_{3}= & -\frac{6}{2 N_{c}+N_{f}} y_{\mu=0}\left\langle\operatorname{Tr} A_{0}^{2}\right\rangle+V \frac{N_{f}^{2}}{9 \pi^{2}}\left\langle\left(\operatorname{Tr} A_{0}^{3}\right)^{2}\right\rangle \\
& +V \frac{9 N_{f}^{2}}{\left(2 N_{c}+N_{f}\right)^{2}} \bar{\mu}^{2} y_{\mu=0}^{2}\left(\left\langle\left(\operatorname{Tr} A_{0}^{2}\right)^{2}\right\rangle-\left\langle\operatorname{Tr} A_{0}^{2}\right\rangle^{2}\right)
\end{aligned}
$$

Thus, the quark number susceptibility is obtained by measuring the condensates $\left\langle\operatorname{Tr} A_{0}^{2}\right\rangle,\left\langle\left(\operatorname{Tr} A_{0}^{2}\right)^{2}\right\rangle$ and $\left\langle\left(\operatorname{Tr} A_{0}^{3}\right)^{2}\right\rangle$ on the lattice. Due to the superrenormalizable nature of the theory, measurements can be rigorously converted to $\overline{\mathrm{MS}}$ scheme in the lattice continuum limit; because $\overline{\mathrm{MS}}$ was used in in the perturbative matching to $4 \mathrm{~d} \mathrm{QCD}$, this also allows us to compare to $4 \mathrm{~d}$ results. For example, the continuum limit for the $\left\langle\left[\operatorname{Tr} A_{0}^{3}\right]^{2}\right\rangle$ is

$$
\left.V\left\langle\left[\operatorname{Tr} A_{0}^{3} / g_{3}^{3}\right]^{2}\right\rangle_{\overline{\mathrm{MS}}}=\lim _{\beta \rightarrow \infty}\left\{V\left\langle\left[\operatorname{Tr} A_{0}^{3} / g_{3}^{3}\right]^{2}\right]\right\rangle_{a}-\frac{5}{16 \pi^{2}}[\ln \beta+0.08848010]\right\},
$$

where $\beta \equiv \frac{2 N_{c}}{a g_{3}^{2}}$ is the lattice coupling constant. Continuum limits for $\left\langle\operatorname{Tr} A_{0}^{2}\right\rangle$ and $\left\langle\left(\operatorname{Tr} A_{0}^{2}\right)^{2}\right\rangle$ are given in [11]. The relation between $\chi_{3}$ and the true $4 \mathrm{~d}$ susceptibility is

$$
\chi=\frac{g_{3}^{6}}{T^{3}} \chi_{3}+\frac{\partial^{2}}{\partial \mu_{u}^{2}} \Delta p
$$

where $\Delta p=p_{\mathrm{QCD}}-p_{3 \mathrm{~d}}$ is the perturbative $3 \mathrm{~d} \rightarrow 4 \mathrm{~d}$ matching coefficient, and can be found in [6].

\section{Lattice measurements}

Lattice simulations were carried out for $N_{\mathrm{f}}=2$. We used large lattice sizes $V=140^{3}, 200^{3}$ and values of $\beta=32,40,67,80,120$. The volumes are large enough so that the remaining (exponential) finite volume effects are safely below our statistical accuracy; for a detailed analysis in a related theory see [12].

For each value of $\beta$ we did simulations with values of $y=6.62,5.31,4.00,3,09,2.02,1.18$, 0.71 , and 0.45 . The simulations have been carried out for $\mu=0$, and to obtain the susceptibility, one needs measurements of $\left\langle\operatorname{Tr} A_{0}^{2}\right\rangle$ and $\left\langle\left(\operatorname{Tr} A_{0}^{3}\right)^{2}\right\rangle$. 

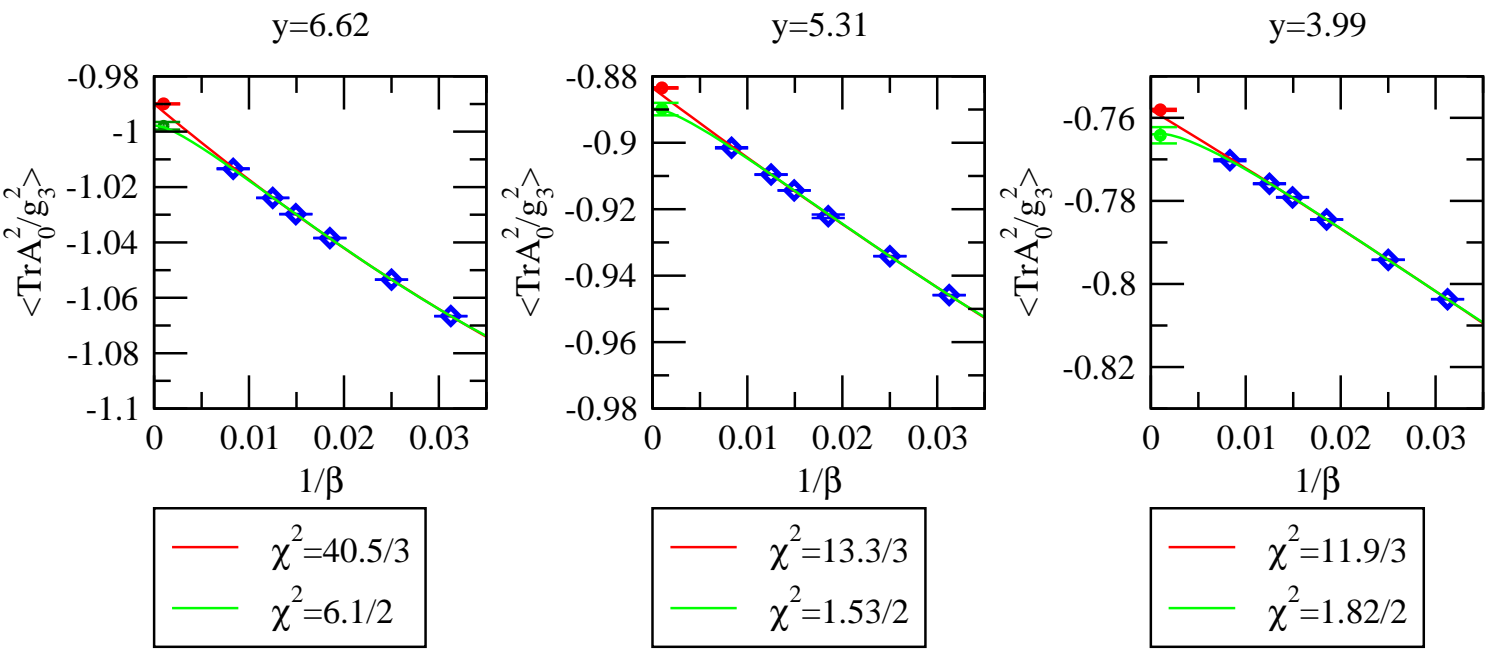

Figure 1: Difference of continuum extrapolations with and without the log-term. The solid line is a fit $c_{1}+\frac{c_{2}}{\beta}+\frac{c_{3}}{\beta^{2}}$ and the dashed line $c_{1}+\frac{c_{2}}{\beta}+\frac{c_{2}^{\prime}}{\beta} \log (\beta)+\frac{c_{3}}{\beta^{2}}$.

The standard procedure of doing continuum extrapolation is to fit a polynomial to the divergence subtracted lattice data. However, in EQCD the divergent lattice contributions contain also terms of the form $\beta \log (\beta)$ and such terms could also arise in terms $\sim a \sim 1 / \beta$. Therefore, we do continuum extrapolation in two ways; we use a second order polynomial

$$
c_{1}+\frac{c_{2}}{\beta}+\frac{c_{3}}{\beta^{2}},
$$

and a fitting function of the form

$$
c_{1}+\frac{c_{2}}{\beta}+\frac{c_{2}^{\prime}}{\beta} \log (\beta)+\frac{c_{3}}{\beta^{2}} .
$$

The Fig. 1 demonstrates the differences between different continuum extrapolations for $\left\langle\operatorname{Tr} A_{0}^{2}\right\rangle$. The extrapolations done using (3.2) have excellent $\chi^{2} /$ dof, and we shall use this form henceforth. The full continuum extrapolations are shown in Fig. 2. We note that the detailed form of the fitting function is significant for our results; thus, knowledge of the true $1 / \beta$ coefficient is highly desirable. There is an ongoing calculation of this term using stochastic perturbation theory [13], which will hopefully confirm our result.

The contributions of $\left\langle\left(\operatorname{Tr} A_{0}^{3}\right)^{2}\right\rangle$ to susceptibility are numerically much smaller than that of $\left\langle\operatorname{Tr} A_{0}^{2}\right\rangle$. The measurements of $\left\langle\left(\operatorname{Tr} A_{0}^{3}\right)^{2}\right\rangle$ are not accurate enough to distinguish between extrapolations (3.1) and (3.2), and the choice is not significant for the final results. Hence we use the second order function in Fig. 2.

The continuum extrapolated results are shown in Fig. 3, as a function of the parameter $y(T)$. The result agrees very well with the perturbative susceptibility derived in ref. [14]. The perturbative result has the form:

$$
\chi_{\text {pert }}=a_{1} y^{3 / 2}+a_{2} y+a_{3} y^{1 / 2}+a_{4}
$$



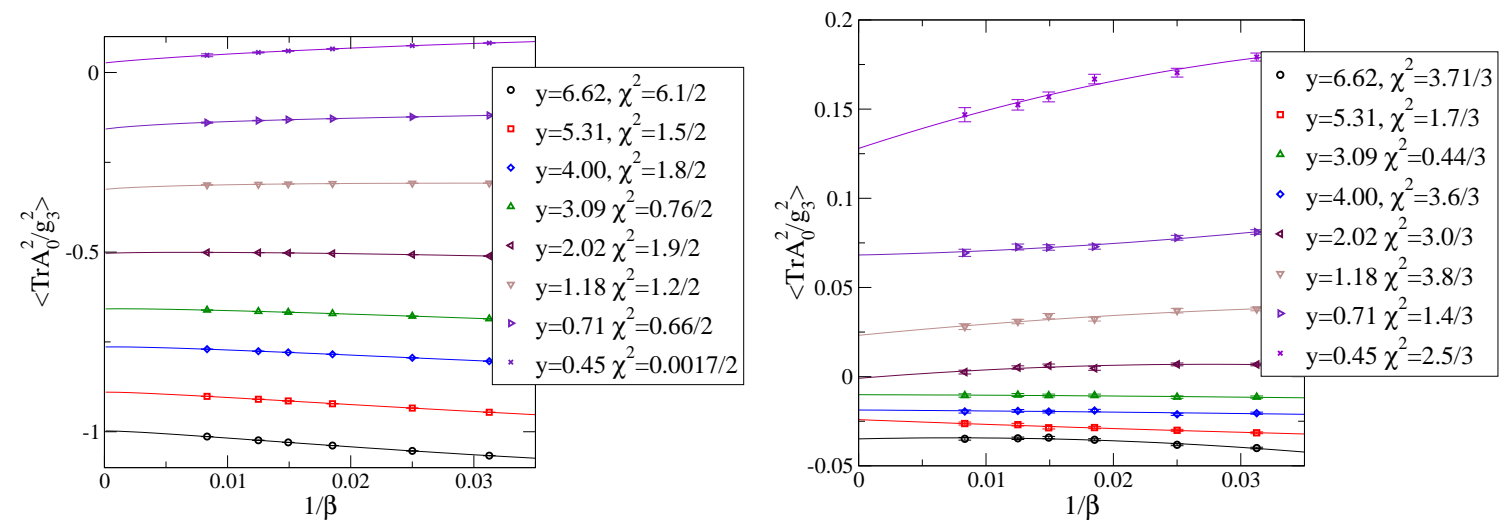

Figure 2: Continuum limits of $\left\langle\operatorname{Tr} A_{0}^{2}\right\rangle$ and $\left\langle\left(\operatorname{Tr} A_{0}^{3}\right)^{2}\right\rangle$ at different values of $y$.
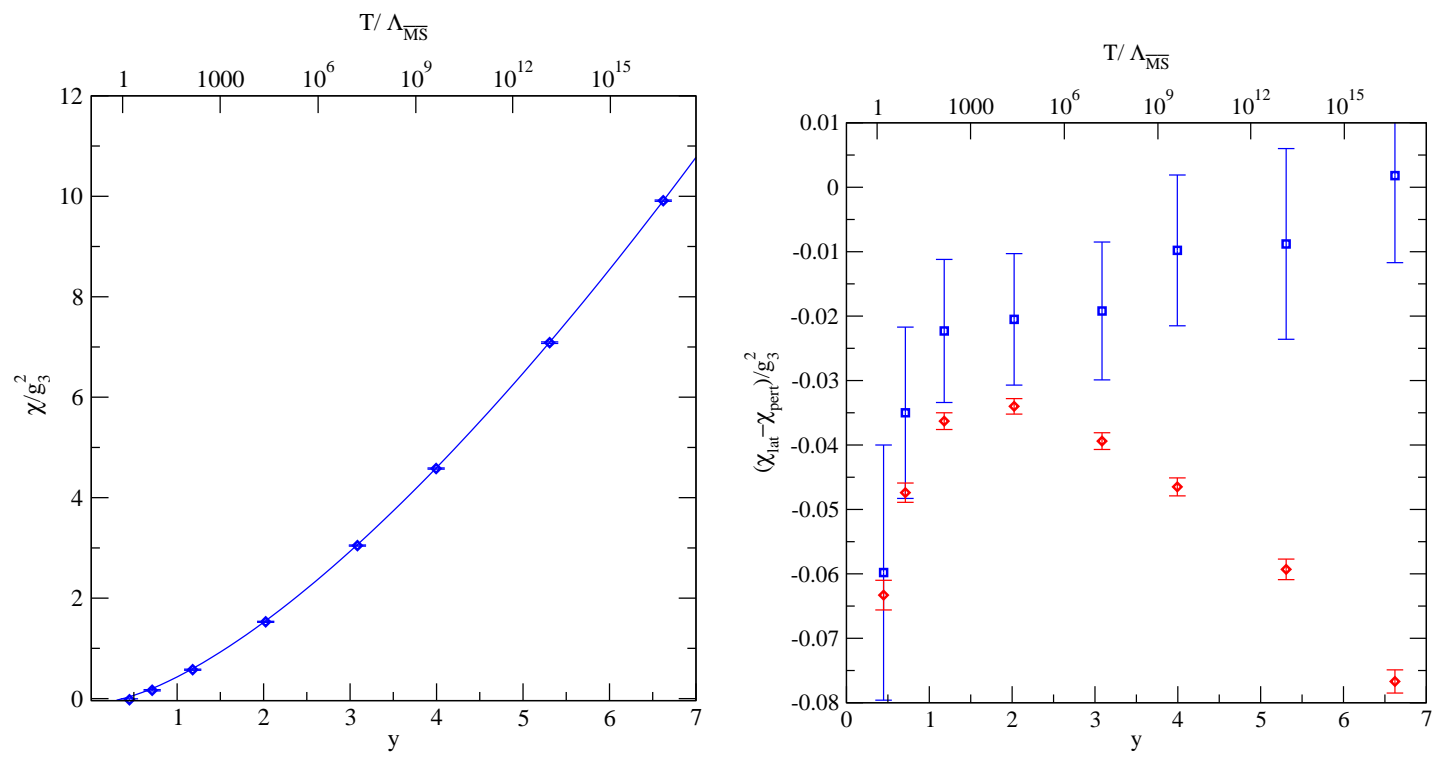

Figure 3: Left: the susceptibility as a function of $y$ and $T / \Lambda_{\overline{\mathrm{MS}}}$. The solid line is the perturbative prediction. Right: the difference between the perturbation theory and lattice results. Points denoted by squares are obtained from logarithmic continuum extrapolation and diamonds from polynomial extrapolation; the former one has expected behavior as $y \rightarrow \infty$.

where $a_{i}$ are functions of $x$ and $z$. On the right panel we show the difference between the perturbative result and the lattice measurement. Because the lattice measurement (in the continuum limit) fully includes perturbative contributions, we expect the difference to behave as $O\left(y^{-1 / 2}\right)$. This is the case when continuum extrapolation is performed using Eq. (3.2); the extrapolation (3.1) diverges from perturbative result as $y \rightarrow \infty$, which is inconsistent. This also motivates the use of (3.2) as continuum extrapolation.

In Fig. 4 we show the susceptibility in $4 \mathrm{~d}$ units, transformed using Eq. (2.7), and compare the result to $4 \mathrm{~d} N_{\mathrm{f}}=2$ staggered fermion lattice simulations $[16,17]$. Here we use the ratio $T_{c} / \Lambda_{\overline{\mathrm{MS}}}=$ 0.49 [18]. We can observe that our results agree very well with $4 \mathrm{~d}$ simulations and with perturbation 


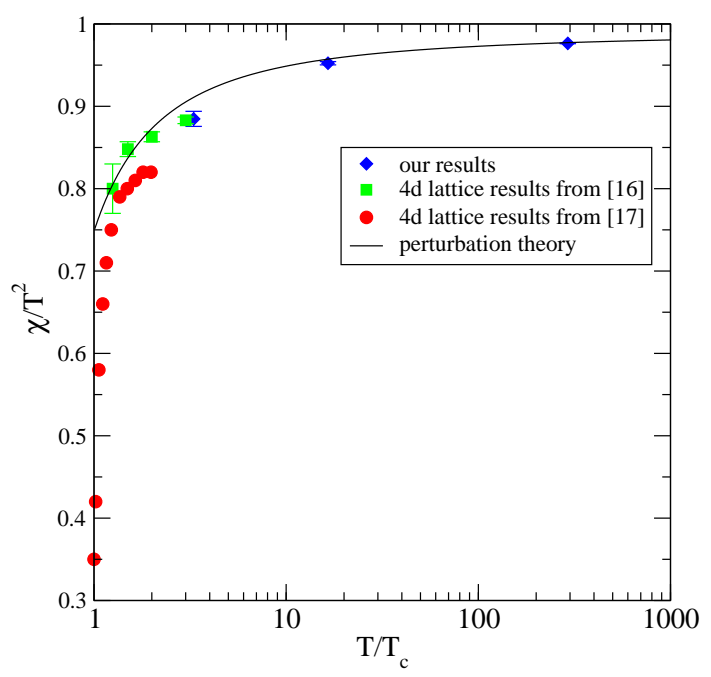

Figure 4: The quark number susceptibility in $4 \mathrm{~d}$ units. Our results are shown with diamonds, only 3 points fit in the temperature range shown. The results agree very well with $4 \mathrm{~d}$ lattice simulation results $[16,17]$.

theory. Indeed, the difference between EQCD measurements and perturbative results are all but invisible on the scale of the plot, possibly excluding the lowest temperature data.

\section{Conclusions and Outlook}

We have measured the quark number susceptibility in dimensionally reduced effective theory of high- $T$ QCD. The results show a good agreement with the perturbation theory and $4 \mathrm{~d}$ lattice simulations, down to temperatures $\sim 3 T_{c}$. Currently we are expanding our simulations for finite chemical potential. In order to avoid the sign problem at finite $\mu$ we do the simulations using imaginary chemical potential and then continue analytically to real values.

\section{Acknowledgments}

This work was partly supported by the Magnus Ehrnrooth Foundation, a Marie Curie Host Fellowship for Early Stage Researchers Training, and Academy of Finland, contract number 104382. Simulations were carried out at Finnish Center for Scientific Computing (CSC).

\section{References}

[1] P. H. Ginsparg, First Order And Second Order Phase Transitions In Gauge Theories At Finite Temperature, Nucl. Phys. B 170 (1980) 388.

[2] T. Appelquist and R. D. Pisarski, High-Temperature Yang-Mills Theories And Three-Dimensional Quantum Chromodynamics, Phys. Rev. D 23 (1981) 2305.

[3] K. Kajantie, M. Laine, K. Rummukainen and M. E. Shaposhnikov, Generic rules for high temperature dimensional reduction and their application to the standard model, Nucl. Phys. B 458 (1996) 90 [hep-ph/9508379]. 
[4] E. Braaten and A. Nieto, Free Energy of QCD at High Temperature, Phys. Rev. D 53 (1996) 3421 [hep-ph/9510408].

[5] K. Kajantie, M. Laine, K. Rummukainen and Y. Schröder, The pressure of hot QCD up to $g^{6} \ln (1 / \mathrm{g})$, Phys. Rev. D 67 (2003) 105008 [hep-ph/0211321].

[6] A. Vuorinen, The pressure of QCD at finite temperatures and chemical potentials, Phys. Rev. D 68 (2003) 054017 [hep-ph/0305183].

[7] A. Gynther and M. Vepsalainen, Pressure of the standard model at high temperatures, JHEP 0601, 060 (2006) [hep-ph/0510375].

[8] K. Kajantie, M. Laine, K. Rummukainen and Y. Schroder, How to resum long-distance contributions to the QCD pressure? Phys. Rev. Lett. 86 (2001) 10 [arXiv:hep-ph/0007109].

[9] M. Laine and Y. Schroder, Two-loop QCD gauge coupling at high temperatures, JHEP 0503 (2005) 067 [arXiv:hep-ph/0503061].

[10] A. Hart, M. Laine and O. Philipsen, Static correlation lengths in QCD at high temperatures and finite densities, Nucl. Phys. B 586 (2000) 443 [hep-ph/0004060].

[11] K. Kajantie, M. Laine, K. Rummukainen and M. E. Shaposhnikov, $3 d S U(N)+$ adjoint Higgs theory and finite-temperature QCD, Nucl. Phys. B 503 (1997) 357 [hep-ph/9704416].

[12] A. Hietanen, K. Kajantie, M. Laine, K. Rummukainen and Y. Schroder, Plaquette expectation value and gluon condensate in three dimensions, JHEP 0501 (2005) 013 [hep-lat/0412008].

A. Hietanen and A. Kurkela, Plaquette expectation value and lattice free energy of three-dimensional $S U(N(c)$ gauge theory, hep-lat/0609015.

[13] C. Torrero, M. Laine, Y. Schroder, F. Di Renzo and V. Miccio, Renormalization of infrared contributions to the QCD pressure, PoS(LAT2006)038 [hep-lat/0609048].

[14] A. Vuorinen, Quark number susceptibilities of hot QCD up to $g * * 6 \ln (g)$, Phys. Rev. D 67 (2003) 074032 [hep-ph/0212283].

[15] A. Hart, M. Laine and O. Philipsen, Testing imaginary vs. real chemical potential in finite-temperature QCD, Phys. Lett. B 505 (2001) 141 [hep-lat/0010008].

[16] R. V. Gavai, S. Gupta and P. Majumdar, Susceptibilities and screening masses in two flavor QCD, Phys. Rev. D 65 (2002) 054506 [hep-lat/0110032].

[17] F. Karsch, S. Ejiri and K. Redlich, Hadronic fluctuations in the QGP, Nucl. Phys. A 774 (2006) 619 [hep-ph/0510126].

[18] S. Gupta, A precise determination of T(c) in QCD from scaling, Phys. Rev. D 64 (2001) 034507 [hep-lat/0010011]. 